\title{
Copper Chaperone for Superoxide Dismutase Expression is Down-Regulated and Correlated with More Malignant Tumoral Features and Poor Prognosis in Human Hepatocellular Carcinoma
}

\section{Chun-yong Wen}

Sun Yat-sen University First Affiliated Hospital

Chang-liang Shan

Nankai University

Wen-jing Sun

Jinan University

Yuan Wan

Sun Yat-sen University First Affiliated Hospital

Run Lin

Sun Yat-sen University First Affiliated Hospital

Bin Chen

Sun Yat-sen University First Affiliated Hospital

Hai-tao Dai

Sun Yat-sen University First Affiliated Hospital

Ke-yu Tang

Sun Yat-sen University First Affiliated Hospital

Xian-hong Xiang

Sun Yat-sen University First Affiliated Hospital

Jian-yong Yang

Sun Yat-sen University First Affiliated Hospital

Nan Li

Guangzhou First People's Hospital

Huang Yonghui ( $\nabla$ hyongh@mail.sysu.edu.cn )

Sun Yat-sen University First Affiliated Hospital https://orcid.org/0000-0001-5921-583X

\section{Research}

Keywords: Hepatocellular carcinoma, Copper chaperone for superoxide dismutase, Malignant biomarkers, Prognosis, Bioinformatic analysis 
Posted Date: October 25th, 2021

DOI: https://doi.org/10.21203/rs.3.rs-100249/v2

License: (c) (i) This work is licensed under a Creative Commons Attribution 4.0 International License. Read Full License 


\section{Abstract}

Background: Up-regulated expression of copper chaperone for superoxide dismutase (CCS) is identified in a multitude of tumors and is closely related to more malignant tumoral biological behaviors. However, little is known about the role of CCS in hepatocellular carcinoma (HCC). This study aims to explore the expression pattern and the significance of CCS in human HCC.

Methods: Fresh samples of HCC with paired adjacent tissues were obtained from 32 patients who underwent hepatectomy. The expression levels of CCS and representative malignant biomarkers in HCC were investigated by western blotting and immunohistochemistry staining, respectively. The correlation between the expression status of CCS and the activities of selected malignant biomarkers and important drive genes of HCC oncogenesis, patient's clinicopathological features and prognosis, were analyzed. Additional bioinformatics investigation of dataset retrieved from database were performed to further explore the role of CCS in human HCC.

Results: The majority of the HCC tumor $(78.1 \%, 25 / 32)$ presented a lower expression of CCS, whereas in the minority of the tumor $(22.9 \%, 7 / 32)$ the expression of CCS was determined as higher. Furthermore, it was found that the expression level of CCS was significantly correlated with malignant biomarkers. The expression of CCS was reversely correlated with ES grade and TNM stage to some extent. And there is a significant correlation between the CCS gene and a series of genes in the development and progression of HCC. Patients with higher CCS expression level were prone to have a better prognosis.

Conclusion: HCC presented a unique lower expression pattern of CCS which reversely correlated with the more malignant tumoral features and poor prognosis.

\section{Background}

The management of HCC remains a great challenge that the majority of the patients cannot be diagnosed at early stage when radical treatments are feasible[1]. Furthermore, it is unfortunately that only a minimal survival benefit can be achieved by currently available systemic therapies[2]. There is an urgent need to explore novel theranostic targets and biomarkers to improve the prognosis. To achieve this goal, elucidating the biological alterations and related genes which regulate the signal pathways involved in the occurrence and development of HCC is prerequisite. Of them, it is well known that the "Warburg effect" is one of the most unique and important adaptions to fulfill the substantial increment in energy demand for tumor survival, rapid proliferation and invasion[3]. Meanwhile, reactive oxygen species (ROS), a sort of substances with contradictory roles is produced at high a concentration during aerobic glycolysis[4]. Therefore, how the tumor cells balance the pros and cons of ROS is a critical factor to determine the cell fate[5]. As a key regulator, copper chaperone for superoxide dismutase (CCS) is vitally important for tumor cells to handle ROS[6-8]. The expression of CCS was reported to be considerably increased in the vast majority of investigated tumor types which was closely correlated with more aggressive malignant biological behaviors and impaired patient prognosis $[9,10]$. More importantly, it has been proven that 
targeting CCS to modulate its expression pattern is conducive to the treatment of certain types of tumor $[9,10]$. These findings implied that CCS could potentially be a biomarker and target for the management of tumors. However, rare focus has been placed on the role of CCS in HCC.

In this study, the expression pattern and significance of CCS in human HCC were explored using surgically resected samples. The correlations between CCS and a set of selected malignant biomarkers, clinicopathological factors, and patient prognosis were investigated. To validate the experimental findings, bioinformatic analyses were performed based on data of TCGA and HPA.

\section{Materials And Methods}

\section{Patients and specimens}

This study was approved by the Ethics Committee of the First Affiliated Hospital, Sun Yat-sen University ([2019] 365) and complied with the 1964 Declaration of Helsinki and its later amendments or comparable ethical standards. Fresh samples (Fig S1) of HCC tumor mass (T) with paired para-carcinoma tissues (P1: $\leq 1 \mathrm{~cm}$ and P2: $1-5 \mathrm{~cm}$, distance to tumor, if available) and normal liver tissues ( $>5 \mathrm{~cm}$ distance to tumor, if available) were obtained from $32 \mathrm{HCC}$ patients who did not received anticancer treatment before surgery and underwent hepatectomy between January 2018 and December 2018 at the First Affiliated Hospital, Sun Yat-sen University. A total of 32 tumoral, 58 para-carcinoma (32 P1 and 26 P2), and 9 normal liver tissue samples were obtained for this study. A series of clinicopathological parameters including age, gender, microvascular invasion (MVI), vascular invasion, etc., were documented (Table 1). Preoperative liver function was assessed by the Child-Pugh scoring system. Tumor differentiation was graded by the Edmondson- Steiner (ES) grading system[11]. The tumor stage was determined according to the sixth edition of the tumor-node-metastasis (TNM) classification of the International Union Against Cancer (UICC) and the Barcelona Clinic Liver Cancer (BCLC) strategy[12, 13]. Tumor size was defined as the maximum diameter of the lesion and the largest one was selected when there were multiple lesions. The follow-up assessment was implemented using hepatic ultrasonography, computed tomography, and Magnetic Resonance Imaging, every month during the first 6 months after resection, and every 3 months thereafter. New lesions that arose in the residual liver in the imaging study were considered to be tumor recurrence.

\section{Table 1. Clinicopathological features of the HCC patients}




\begin{tabular}{|c|c|}
\hline Clinicopathological features & Results \\
\hline Median age (range, years) & $55(29-71)$ \\
\hline Gender (male/female) & $27 / 5$ \\
\hline Hepatitis (positive/negative) & $26 / 6$ \\
\hline Liver cirrhosis (present/absent) & $16 / 16$ \\
\hline Preoperative AFP( $\leq 20>/ 20, \mathrm{ng} / \mathrm{mL} \rrbracket$ & $10 / 22$ \\
\hline Tumor size $(\mathrm{cm})(\leq 5 />5, \mathrm{~cm})$ & $12 / 20$ \\
\hline Tumor nodule number (single/multiple) & $26 / 6$ \\
\hline Child-Pugh classification (A /B) & $26 / 6$ \\
\hline BCLC stage $(A / B / C)$ & $20 / 0 / 12$ \\
\hline 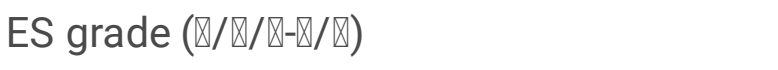 & $0 / 17 / 7 / 8$ \\
\hline MVI (present/absent) & $17 / 15$ \\
\hline Vascular invasion (present/absent) & $9 / 23$ \\
\hline Extrahepatic metastasis (present/Absent) & $4 / 28$ \\
\hline 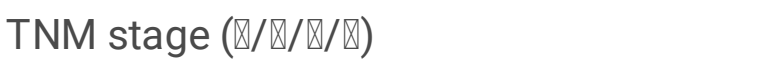 & $22 / 0 / 5 / 5$ \\
\hline
\end{tabular}

\section{Reagents}

Rabbit polyclonal antibody against CCS (Cat. no. 22802-1-AP, 1:1000 dilution), mouse monoclonal antibody against human beta-actin (Cat. no. 66009-1-Ig, 1:3000 dilution), horseradish peroxidaseconjugated goat anti-mouse IgG, and horseradish peroxidase-conjugated goat anti-rabbit IgG were purchased from Proteintech Group (Chicago, IL, USA). Rabbit polyclonal antibody against Ki-67 (Cat. no. ZM-0165, 1/200 dilution), rabbit polyclonal antibody against E-cadherin (Cat. no. ZM-0092, 1/400 dilution), rabbit polyclonal antibody against vimentin (Cat. no. ZM-0260, 1/500 dilution), rabbit polyclonal antibody against GPC3 (Cat. no. ZM-0146, 1/300 dilution), and rabbit polyclonal antibody against CD34 (Cat. no. ZA-0550, 1/400 dilution) were purchased from Beijing Zhongshan Golden Bridge Biotechnology (Beijing, China) for immunohistochemistry (IHC) assay.

\section{Western blot analysis}

Western blotting was performed on samples of HCC tissues, para-carcinoma tissues, and normal liver tissues. The tissues were homogenized on ice in lysis buffer containing PBS, $1.5 \mathrm{~mol} / \mathrm{L} \mathrm{NaCl}, 1.0 \mathrm{~mol} / \mathrm{L}$ HEPES (pH 7.0), $1 \% \mathrm{NP}-40,0.1 \mathrm{~mol} / \mathrm{L} \mathrm{Na}_{4} \mathrm{P}_{2} \mathrm{O}_{7}, 0.1 \mathrm{~mol} / \mathrm{L}$ sodium fluoride, $0.1 \mathrm{~mol} / \mathrm{L}$ sodium orthovanadate, protease inhibitors $(2 \mathrm{mmol} / \mathrm{L}$ phenylmethylsulfonyl fluoride, $10 \mu \mathrm{g} / \mathrm{mL}$ leupeptin, 10 $\mu \mathrm{g} / \mathrm{mL}$ aprotinin) for $30 \mathrm{~min}$. The homogenates were clarified by centrifugation at $12,000 \mathrm{rpm}$ for $15 \mathrm{~min}$ 
at $4^{\circ} \mathrm{C}$ and the protein concentration was measured by the method of Bradford. Equal amounts of protein (25 ug) were separated on $12 \%$ SDS-PAGE and electrophoretically transferred to polyvinylidene fluoride membranes (Millipore, Billerica, MA, USA) using a mini trans-blot (Tanon, Shanghai, China). After skimmilk blocking, the membranes were incubated with rabbit polyclonal antibody against CCS at 1:1000 dilution and mouse monoclonal antibody against human beta-actin at 1:3000 dilution respectively. Membranes were then washed thrice with PBST and incubated with horseradish peroxidase-conjugated goat anti-rabbit IgG at 1:1000 and horseradish peroxidase-conjugated goat anti-mouse IgG at 1:3000 dilution for $1 \mathrm{~h}$ respectively. Signals were detected using Luminol substrate solution. The amount of CCS protein expression was measured semi-quantitatively using the Quantity One software (version 4.6.6, BioRad laboratories, USA) by analyzing the peak area of the CCS protein band relative to the beta-actin within the same sample on the same membrane. The ratio of the gray value of the target protein band to that of the corresponding $\beta$-actin was calculated as the expression level of the target band. The CCS expression levels of the tumor were divided into high and low groups according to their relative expressions as compared to those in the corresponding non-tumor tissues.

\section{Immunohistochemistry examinations}

The expression levels of Ki-67, E-cadherin, vimentin, glypican-3 (GPC3), and microvessel density (MVD, as represented by CD34) in HCC and non-tumoral tissues were assessed by IHC. IHC images were evaluated by two independent observers who were blinded to clinical data and were scored semiquantitatively based on staining intensity and distribution according to the immunoreactive score (IRS) scale which

took the percentage of positive cells (scale 0 to 4 ) and the intensity of the color reaction (scale 0 to 3 ) into account[14]. The values of positive percentage of counted cells were scored as follows: 0-point, no positive cells; 1-point, < 10\%; 2-points, 10-50\%; 3-points,51-80\%; and 4-points, $>80 \%$, respectively. And the values of intensity of color reaction were scored as follows: 0 -point, no reaction; 1-point, weak color reaction; 2-points, moderate color reaction; and 3-points, strong color reaction, respectively. The final score ranged from 0 to 12 points which was calculated by multiplying the value of positive percentage of counted cells to the value of intensity of color reaction. Specifically, the expression level of CD34 was scored semiquantitatively based on the "hot spots" method. Each of the isolated microvascular in the tumor tissue was counted as a "hot spots"[15]. Ten randomly selected images with 40X magnification were used for scoring for each of the biomarker, and 5 images for CD34, specifically.

\section{Bioinformatics analysis}

The gene and protein data of CCS, relevant biomarkers, and selected driver gene mutations of HCC, as well as patient prognosis and clinicopathological features, were retrieved from HPA (https://www.proteinatlas.org/, accessed on 20 May 2021) and TCGA (https://www.cancer.gov/, accessed on 20 May 2021) database. The software included in GEPIA (http://gepia.cancer-pku.cn/, accessed on 20 May 2021) and KM plotter (https://kmplot.com/analysis/, accessed on 20 May 2021) was used for data processing and data visualization. The correlations between the CCS expression level 
(high/low) and the aforementioned parameters including relevant biomarkers, driver gene mutations, patient prognosis, and clinicopathological features, were investigated.

\section{Statistical analysis}

All statistical analyses were conducted using SPSS 25.0 statistical software. The expression level of biomarkers between high CCS expression group and low CCS expression group was analyzed using twosided $t$ test. $P<0.05$ was considered as statistically significant difference.

\section{Results}

\section{Expression pattern of CCS in HCC and normal tissues}

The results showed a very unique expression status that the CCS was down-regulated in HCC tissues as compared with adjacent tissues in $78 \%(25 / 32)$ of the patients (Fig. 1a, 1c) while that was elevated in $22 \%(7 / 32)$ of the patients (Fig. 1b, 1c). In order to obtain an overall understanding and comparation of the expression situations of CCS in normal tissues, organs, and the HCC, explorations on the dataset retrieved from HPA and TCGA were conducted. The most intensified CCS gene expression was found in the liver among normal tissues and organs, and there was no statistically significant between the expression strength of CCS gene in the liver and HCC (Fig. 4a).

\section{Expression of CCS, malignant biomarkers, and drive genes in HCC}

The HCC lesions and the patients were divided into high expression group and low expression group according to the western blot results comparing the CCS expression status in tumors and adjacent tissues. IHC was performed on HCC tissues to determine expression levels and difference of Ki-67, Ecadherin, vimentin, GPC3, and CD34 between two groups with different expression level of CCS and their correlations to CCS expression level were shown in Figure 2. The results indicated that the expression levels of Ki67, vimentin, and GPC3 in group with low CCS expression was remarkably higher than those in group with high CCS expression ( $t=3.279, p=0.003 ; t=4.316, p=0.000 ; t=2.988$, $p=0.007$, respectively), whereas the E-cadherin was down-regulated in group with low CCS expression $(t=-2.852, p=0.010)$. There was no significant difference in CD34 expression $(t=-0.306, p=0.786)$ between these two groups. Similar outcomes were observed in when analyzing the data of the TCGA database, with the exception of E-cadherin, which further validated the finding in IHC study (Fig. 2).

Extended analysis displayed strong correlations between CCS expression and most of the included important driving genes in oncogenesis of HCC including TP53, CTNNB1, AXIN1, KEAP1, and RB1, etc. (Fig. 4b, Excel S1)[16, 17]. On the contrary, such relationships were rarely found in normal tissues (Excel S1). Similar outcomes were obtained with respect to the correlations between SOD1 expression and these genes in HCC (Fig S2, Excel S1). The specific $P$ and R values were shown in Excel S1 and correlation between CCS and SOD1gene was shown in Fig S3.

\section{Expression of CCS and clinicopathological features}


The associations between the expression level of CCS and clinicopathological characteristics were listed in Table S1. The results showed that the expression levels of CCS are related to ES grade to some extent $(p=0.15$, Table S1). However, the expression levels of CCS were not significantly correlated with the remaining features including gender, hepatitis $B$ virus infection, liver cirrhosis, preoperative serum AFP level, tumor size, tumor nodule number, Child-Pugh classification, BCLC stage, MVI, vascular invasion, extrahepatic metastasis, and TNM stage. The statistical results from the TCGA database also exhibited same tendency that the CCS expression in HCC of ES $\otimes$ and TNM stage IVB tended to be lower as compared with those in HCC of early ES and TNM stages ( $p=0.146$ and $p=0.071$, respectively, Fig. 4 c).

\section{Expression of CCS and patient prognosis}

Regular follow-up was performed on all of the 32 patients and the median follow-up was 15.8 months (range 0-30 months). Data of three patients were excluded for prognostic study (Fig. 3). due to tumor residue after surgery $(n=1)$, died of liver failure after surgery $(n=1)$, and died of traffic accident $(n=1)$, all of whom were in the low CCS expression group. In CCS high expression group, 42.8\% (3/7) patients relapsed during follow-up and all of the recurrence occurred within 6 months. In CCS low expression group, $54.5 \%$ (12/22) of the patient suffered from tumor relapse. Specifically, $75 \%(9 / 12)$ of the tumor relapse occurred within 6 months post-surgery whereas $25 \%(3 / 12)$ raised over 6 months. Overall, no statistically significant differences could be confirmed regarding the recurrence rate and OS between the CCS high and low expression groups. However, when investigating the dataset of $364 \mathrm{HCC}$ patients which were collected from the KM plotter database, a conclusion can be drawn that patients with high CCS expression $(n=87)$ have a superior prognosis over those with low CCS expression $(n=278)(71.0$ months versus 38.3 months, $p=0.0042$, Fig. 4 d).

\section{Discussion}

Deciphering the biological changes that render the tumor cells with competitive advantages is the prepositive step for developing novel strategies of managements. Copper chaperone for superoxide dismutase (CCS) is an essential regulator in the strict control of the copper metabolism that participates in a broad spectrum of physiological processes, particularly in the homeostasis of ROS[18]. Together with superoxide dismutase 1 (SOD1), CCS modulates the processing procedure of ROS which is produced abundantly during aerobic glycolysis ("Warburg effect") of the tumor cells[6-8]. It has been reported that the CCS expression was substantially reinforced in a plenty of different cancer type, i.e., breast cancer, and further activated subsequent and relevant signaling pathways that promoted tumor progression[9, 10]. Increased CCS expression was a biomarker for more aggressive bio-behavior and poor prognosis. Furthermore, CCS was proven to be a therapeutic target that modulating the CCS activity could suppress the tumor progression. However, little is known with regard to the expression pattern and the significance of CCS in human hepatocellular carcinoma (HCC).

In the current study, it was found that the expression level of CCS was down-regulated in the majority of the HCC. This was a unique expression pattern which is very distinct to tumors originate from other sites. 
The results of TCGA database analysis also showed that the expression of CCS in liver cancer was lower as compared with normal liver tissues. As a critical component of oxidative metabolism, up-regulated CCS prevents excessive accumulation of ROS. The down-regulated CCS expression suggested an apparent different biological adaption of HCC, either HCC cells are more tolerant to the harmful effects of high ROS concentration, or they metabolize ROS through other ways.

IHC examinations were performed in HCC tumor tissues to determine the expression intensity of Ki67, Ecadherin and vimentin, GPC3, and CD34, all of which were well-established biomarkers and prognostic parameters for $\mathrm{HCC}[19]$. Among them, Ki67 is a robust marker for cell propagation, E-cadherin and vimentin are for metastasis, GPC3 is for degree of malignancy, and CD34 is for angiogenesis of the HCC, respectively. The up-regulated of Ki67, vimentin, and GPC3, along with the down-regulated of E-cadherin, in the group of lower CCS expression, indicated these lesions were of more malignant with a higher risk of metastasis and faster growth, and poorer prognosis thereof. Similar relationships were identified the bioinformatic studies. We further surveyed the relationships of CCS expression and a number of core genes in carcinogenesis of $\mathrm{HCC}[16,17]$. It was found that CCS expression became decrease when many of the essential regulatory genes shift toward tumorigenesis and tumor progression. This result implied that suppress of CCS may in favor of HCC development. However, whether they are causal or parallel is not yet known and the exact mechanism of this phenomenon is worthy of further study.

On the correlations between the CCS expression level and clinicopathological characteristics, in spite of both experimental and database results indicated a certain tendency, in particular the ES and TNM stages, but the statistical significance has not reached. Therefore, no definite conclusion can be drawn currently on this issue.

With respect to prognosis, in general, there was a trend of differences in tumor relapse between high $(42.8 \%, 3 / 7)$ and low $(54.5 \%, 12 / 22)$ CCS expression groups. Scrutinization of the detailed prognostic information, most of the tumor recurrences $(80 \%, 12 / 15)$ occurred within 6 months after surgery, and the remaining 3 relapses over 6 months post-surgery occurred in the low expression group. No recrudescence was found in the remaining 4 patients of high CCS expression group. It is widely accepted that recurrence is one of the most important risk factors that impair the patient's prognosis. Previous researches have reported that early recurrence of $\mathrm{HCC}$ may came from the primary metastasis, while the late recurrence was more likely to be developed de novo after surgery[20]. However, there was no consensus with respect to the exact threshold that divides these two categories and the definition of the early recurrence across different reports varied from 6 months to 2 years after surgery. In this work, there was less recurrent tumor for HCC with higher CCS expression, especially in cases exceed 6 months post-surgery, despite statistically significant was not achieved which may due to the limited patients included. Nonetheless, bioinformatic analysis using data from KM plotter database revealed a significant better prognosis, in terms of OS, in high CCS expression of patients as compared to those with low expression. It can be speculated, at least to some degree, that high CCS expression is a positive prognostic factor for HCC patients. 


\section{Conclusion}

The CCS expression was down-regulated in the majority of the HCC lesions, which was quite different as compared to tumors originate from other sites. And the lower CCS expression indicated a more aggressive biological tumor type as demonstrated by both IHC and bioinformatic studies. Furthermore, the CCS expression was closely correlated with the activity of a multitude of core drive genes involved in the development and progression of HCC, suggesting the modulation of CCS pathway may plays an important role in these processes. Moreover, patients with high CCS expression tend to benefit a more favorable recurrence-free survival and prognosis. This study revealed distinct metabolic reshaping that aid to our understanding of the biological behavior of human HCC, and CCS could potential be a useful biomarker for the management of HCC.

\section{Declarations}

\section{Acknowledgements}

This study was supported by Guangdong Natural Science Foundation (2020A1515010920).

\section{Authors' contributions}

YHH, RL and CLS designed the study. CYW and WJS performed the experiments. CYW, YW, BC and HTD collected samples of HCC and adjacent liver tissues. YW, NL and KYT were involved in the analysis of experimental data. CYW performed the bioinformatics analyses. CYW, YHH and RL wrote and revised the manuscript. All authors read and approved the final manuscript.

\section{Funding}

Guangdong Natural Science Foundation (2020A1515010920).

\section{Availability of data and materials}

The datasets generated during and/or analyzed during the current study are available from the corresponding author on reasonable request.

\section{Compliance with ethical standards}

\section{Conflict of interest}

The authors declare that they have no conflicts of interest in regard to this study.

\section{Ethics approval}

This study was approved by the Ethics Committee of the First Affiliated Hospital, Sun Yat-sen University ([2019] 365) and complied with the 1964 Declaration of Helsinki and its later amendments or comparable 
ethical standards.

\section{Consent to participate}

All patients signed the written informed consents.

\section{Consent for publication}

All patients signed the written informed consents for publication.

\section{References}

1. Villanueva A. Hepatocellular Carcinoma. N Engl J Med. 2019;380(15):1450-62. doi:10.1056/NEJMra1713263.

2. Yang JD, Hainaut P, Gores GJ, Amadou A, Plymoth A, Roberts LR. A global view of hepatocellular carcinoma: trends, risk, prevention and management. Nat Rev Gastroenterol Hepatol. 2019;16(10):589-604. doi:10.1038/s41575-019-0186-y.

3. Liberti MV, Locasale JW. The Warburg Effect: How Does it Benefit Cancer Cells? Trends BiochemSci. 2016;41(3):211-8. doi:10.1016/j.tibs.2015.12.001.

4. Reczek CR, Chandel NS. The Two Faces of Reactive Oxygen Species in Cancer. In: Jacks T, Sawyers $\mathrm{CL}$, editors. Annual Review of Cancer Biology, Vol 1. Annual Review of Cancer Biology-Series. Palo Alto: Annual Reviews; 2017. p. 79-98.

5. Weinberg F, Ramnath N, Nagrath D. Reactive Oxygen Species in the Tumor Microenvironment: An Overview. Cancers. 2019;11(8):20. doi:10.3390/cancers11081191.

6. Che MX, Wang R, Li XX, Wang HY, Zheng XFS. Expanding roles of superoxide dismutases in cell regulation and cancer. Drug Discov Today. 2016;21(1):143-9. doi:10.1016/j.drudis.2015.10.001.

7. Banci L, Bertini I, Cantini F, Kozyreva T, Massagni C, Palumaa P et al. Human superoxide dismutase 1 (hSOD1) maturation through interaction with human copper chaperone for SOD1 (hCCS). Proc Natl Acad Sci U S A. 2012;109(34):13555-60. doi:10.1073/pnas.1207493109.

8. Suzuki Y, Ali M, Fischer M, Riemer J. Human copper chaperone for superoxide dismutase 1 mediates its own oxidation-dependent import into mitochondria. Nat Commun. 2013;4:9. doi:10.1038/ncomms3430.

9. Li YP, Liang RH, Zhang XY, Wang JY, Shan CL, Liu SP et al. Copper Chaperone for Superoxide Dismutase Promotes Breast Cancer Cell Proliferation and Migration via ROS-Mediated MAPK/ERK Signaling. Front Pharmacol. 2019;10:11. doi:10.3389/fphar.2019.00356.

10. Wang J, Luo C, Shan CL, You QC, Lu JY, Elf SN et al. Inhibition of human copper trafficking by a small molecule significantly attenuates cancer cell proliferation. Nat Chem. 2015;7(12):968-79. doi:10.1038/nchem.2381.

11. Uhlen M, Zhang C, Lee S, Sjostedt E, Fagerberg L, Bidkhori G et al. A pathology atlas of the human cancer transcriptome. Science. 2017;357(6352):660-+. doi:10.1126/science.aan2507. 
12. European Assoc Study L. EASL Clinical Practice Guidelines: Management of hepatocellular carcinoma. J Hepatol. 2018;69(1):182-236.

13. Heimbach JK, Kulik LM, Finn RS, Sirlin CB, Abecassis MM, Roberts LR et al. AASLD guidelines for the treatment of hepatocellular carcinoma. Hepatology. 2018;67(1):358-80. doi:10.1002/hep.29086.

14. Meyerholz DK, Beck AP. Principles and approaches for reproducible scoring of tissue stains in research. Lab Invest. 2018;98(7):844-55. doi:10.1038/s41374-018-0057-0.

15. Kumagai Y, Sobajima J, Higashi M, Ishiguro T, Fukuchi M, Ishibashi K et al. Angiogenesis in Superficial Esophageal Squamous Cell Carcinoma: Assessment of Microvessel Density Based on Immunostaining for CD34 and CD105. Jpn J Clin Oncol. 2014;44(6):526-33. doi:10.1093/jjco/hyu039.

16. Nakagawa H, Fujita M, Fujimoto A. Genome sequencing analysis of liver cancer for precision medicine. Semin Cancer Biol. 2019;55:120-7. doi:10.1016/j.semcancer.2018.03.004.

17. Gao Q, Zhu HW, Dong LQ, Shi WW, Chen R, Song ZJ et al. Integrated Proteogenomic Characterization of HBV-Related Hepatocellular Carcinoma. Cell. 2019;179(2):561-+. doi:10.1016/j.cell.2019.08.052.

18. Fetherolf MM, Boyd SD, Taylor AB, Kim HJ, Wohlschlegel JA, Blackburn NJ et al. Copper-zinc superoxide dismutase is activated through a sulfenic acid intermediate at a copper ion entry site. $\mathrm{J}$ Biol Chem. 2017;292(29):12025-40. doi:10.1074/jbc.M117.775981.

19. Singhal A, Jayaraman M, Dhanasekaran DN, Kohli V. Molecular and serum markers in hepatocellular carcinoma: Predictive tools for prognosis and recurrence. Crit Rev Oncol/Hematol. 2012;82(2):11640. doi:10.1016/j.critrevonc.2011.05.005.

20. Xu XF, Xing H, Han J, Li ZL, Lau WY, Zhou YH et al. Risk Factors, Patterns, and Outcomes of Late Recurrence After Liver Resection for Hepatocellular Carcinoma A Multicenter Study From China. JAMA Surg. 2019;154(3):209-17. doi:10.1001/jamasurg.2018.4334.

\section{Figures}




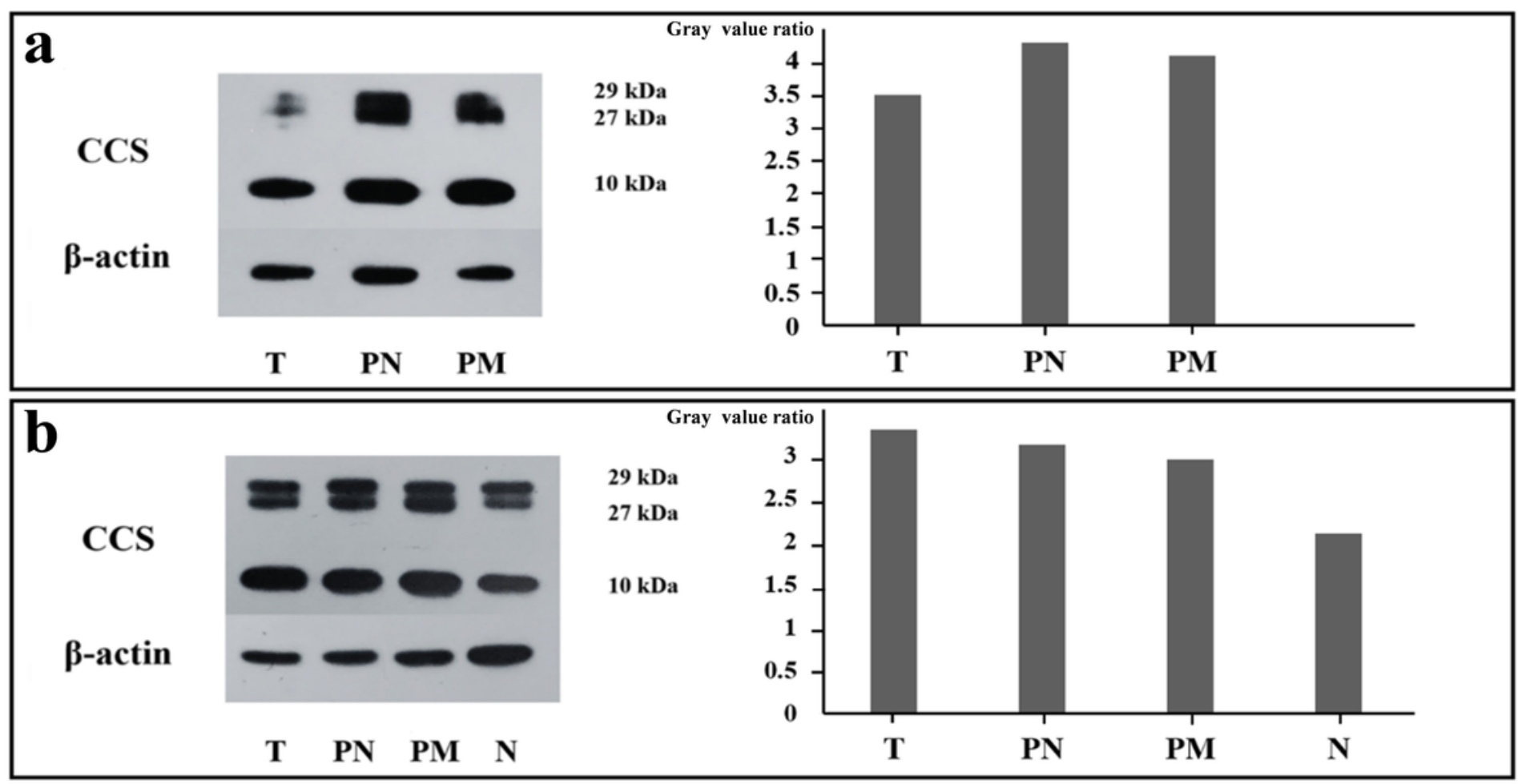

\section{c}

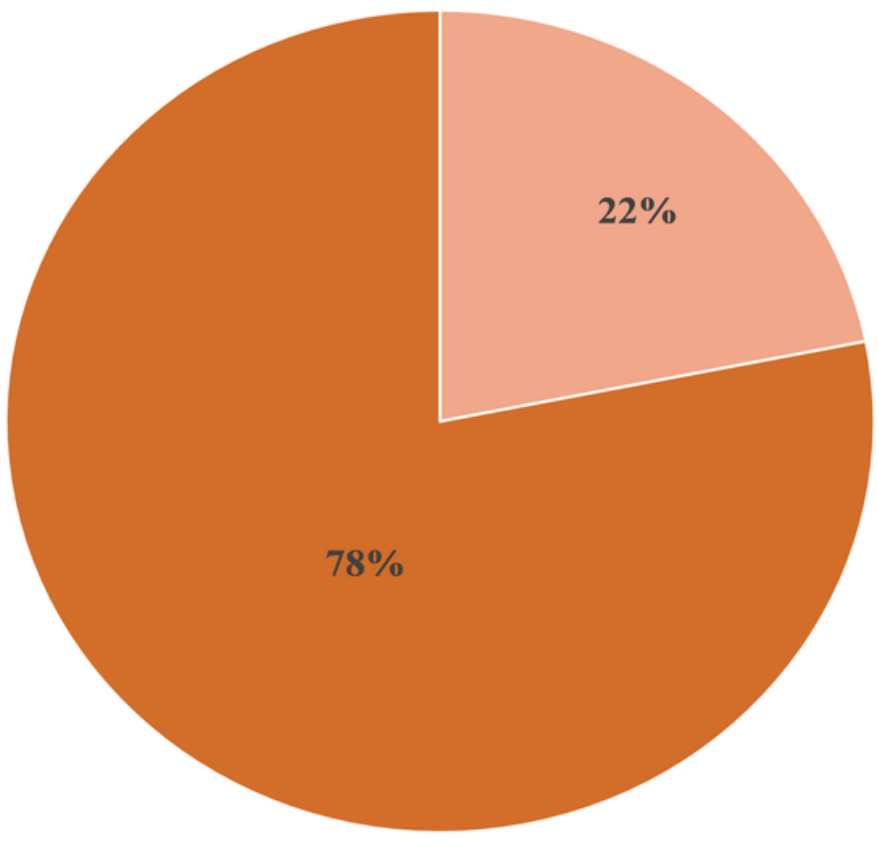

High expression of CCS

Low expression of CCS

Figure 1

Expression of CCS in HCC. Representative western blot results of (a) low and (b) high CCS expression level in HCC tumor mass as compared to that in the counterpart non-tumor tissues. c CCS expression level in 32 patients. Gray value ratio: CCS/ $\beta$-actin. High expression of CCS $\triangle C C S$ expression in HCC is higher than that in adjacent non-cancerous tissues; Low expression of CCS®CCS expression in HCC is lower than that in adjacent non-cancerous tissues. 

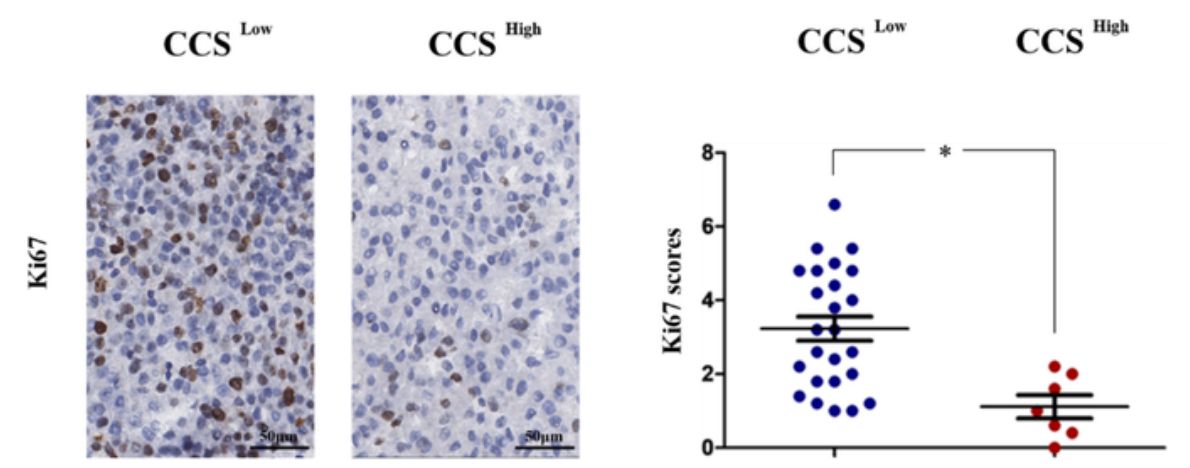

Correlation of related genes
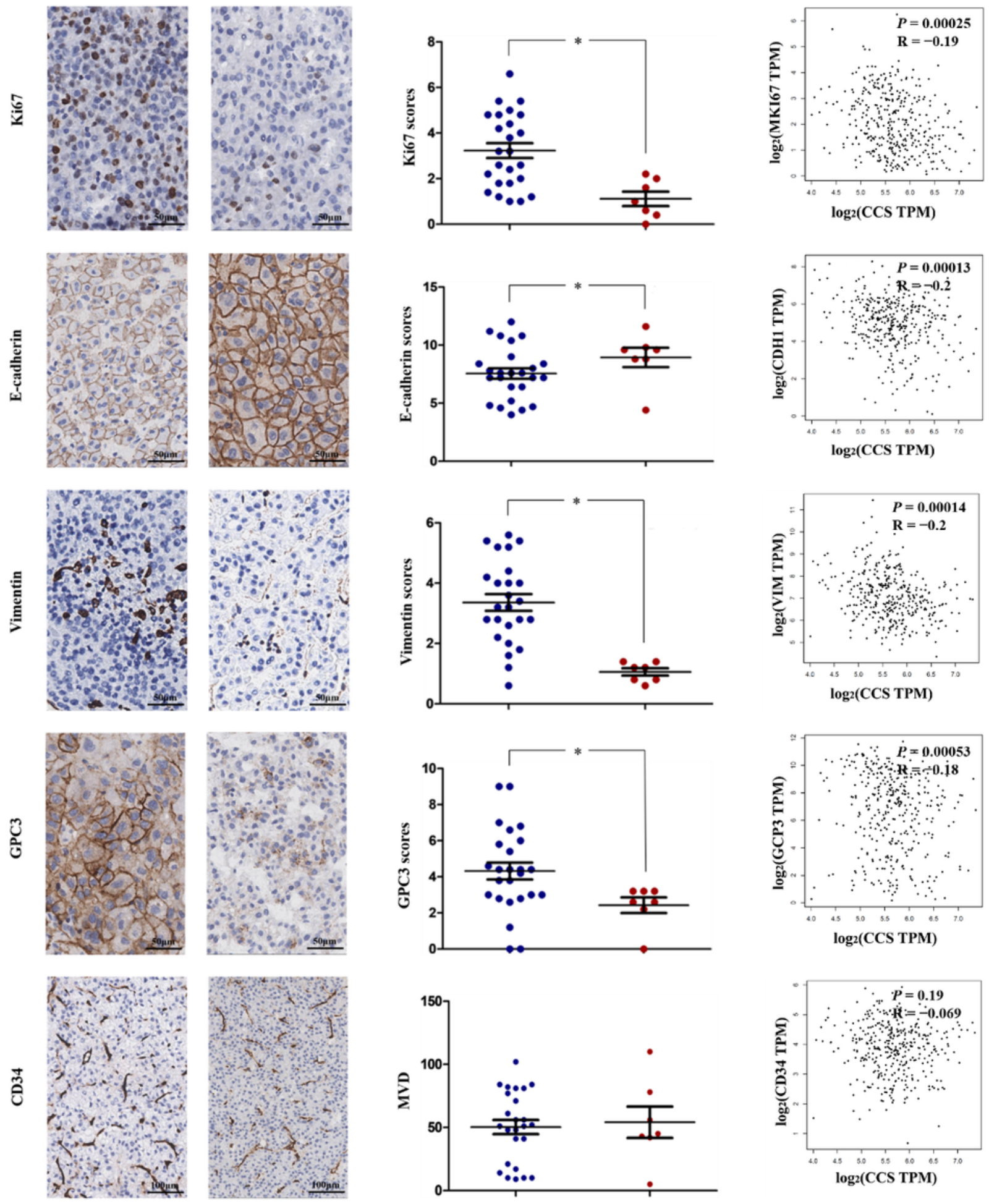

\section{Figure 2}

Representative IHC images, the correlations between expression levels of CCS and selected biomarkers between high and low CCS expression groups, Correlations of expression of CCS gene and that of malignant biomarkers. CCS Low: group with low CCS expression; CCS High: group with high CCS expression. ${ }^{\star} \mathrm{p} \rrbracket 0.05$. 


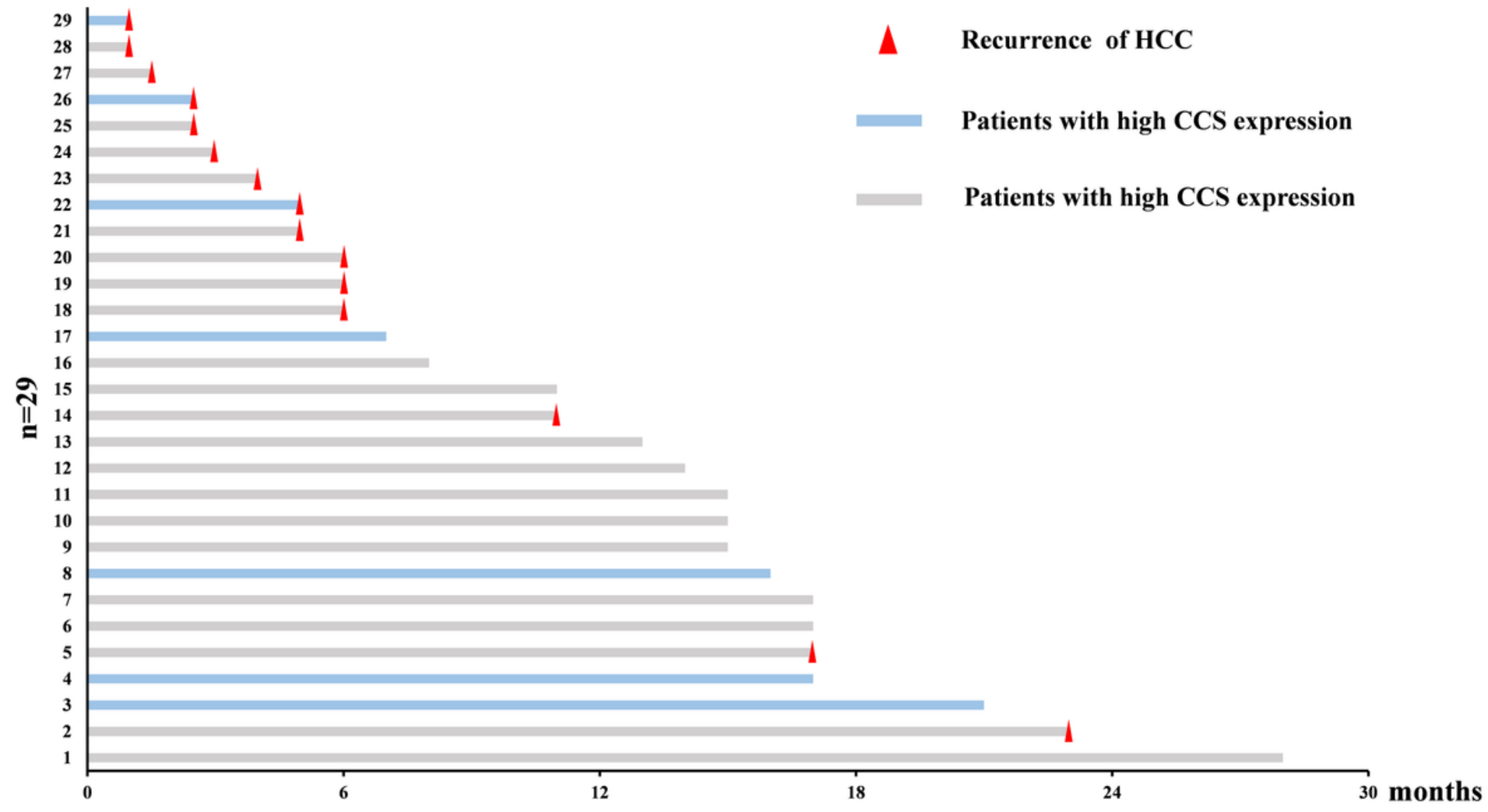

\section{Figure 3}

Expression of CCS and patient prognosis. 
a
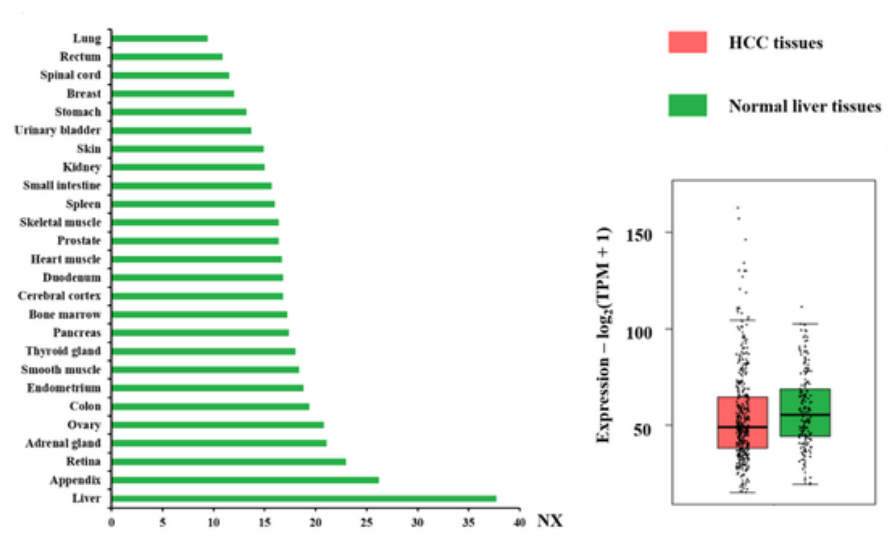

c

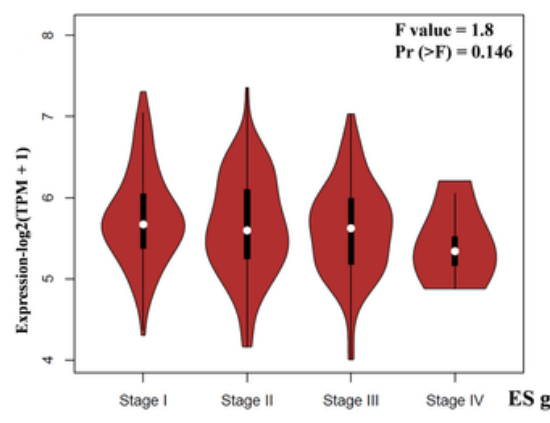

b
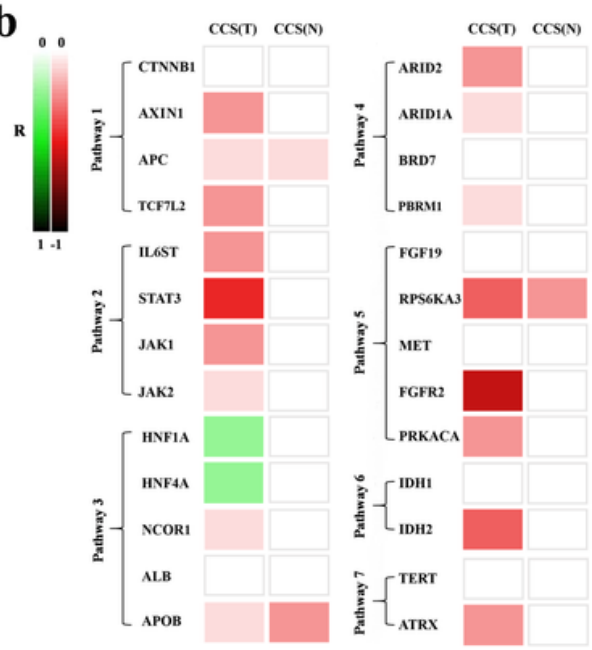

d
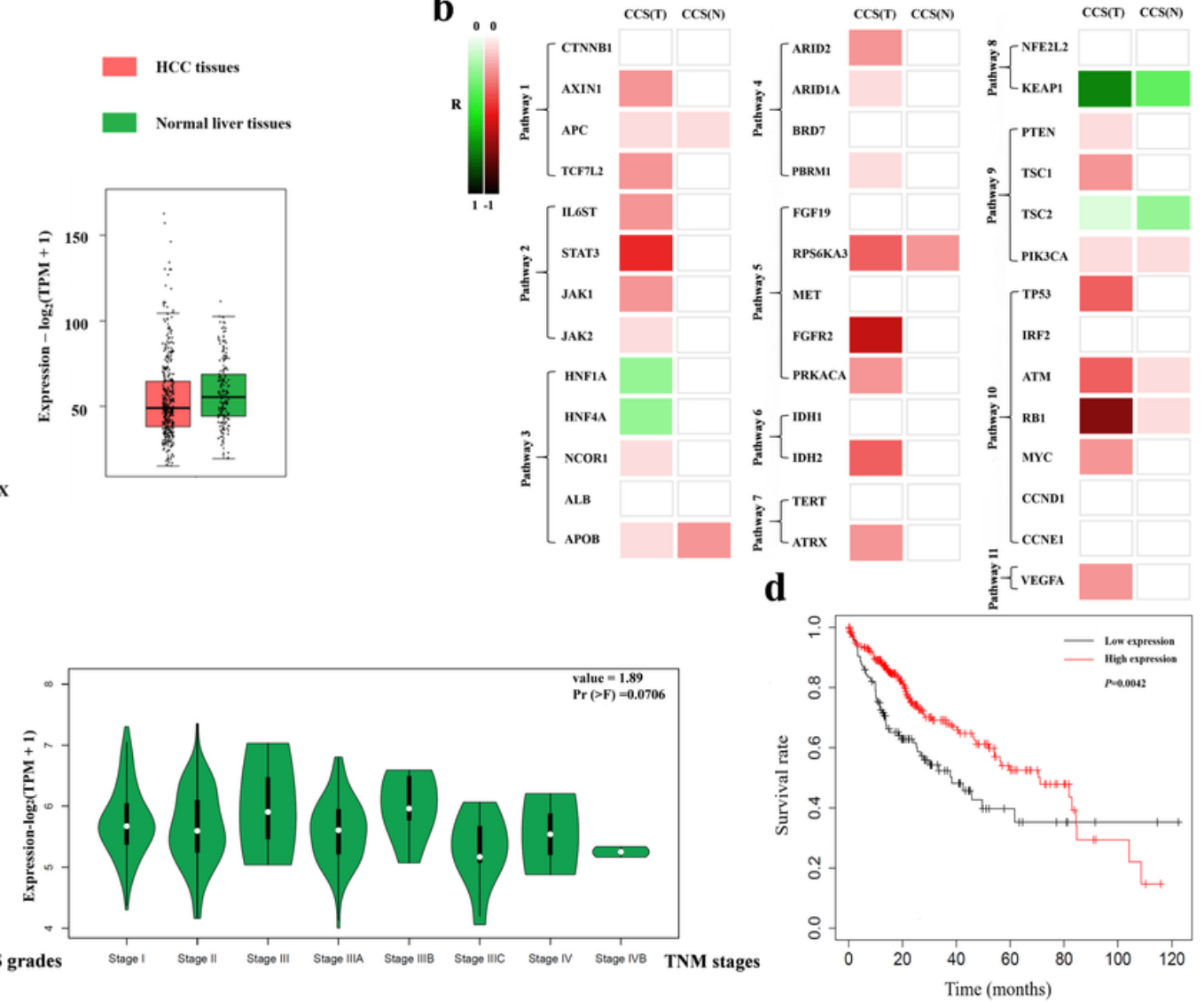

Figure 4

Summary of bioinformatic analysis outcomes. a Expression pattern of CCS in HCC and normal tissues. b Correlations of expression of CCS and drive genes in HCC and normal liver tissues. c Expression of CCS was down-regulated in more advanced stage and aggressive pathological classification of liver cancer. $d$ Low expression of CCS was associated with poor patient prognosis. NX: Normalized expression of gene. Pathway1: Wnt/ß-catenin pathway; Pathway2: Inflammatory pathway; Pathway3: Liver metabolic pathway; Pathway4: Chromatin regulators; Pathway5: Ras/ERK pathway; Pathway6: Matabolism and epigenomics; Pathway7: Telomere maintenace; Pathway8: Oxidative stress pathway; Pathway9: AKT/mTOR pathway; Pathway10: TP53 /cell-cycle pathway; Pathway11: Angiogenesis.

\section{Supplementary Files}

This is a list of supplementary files associated with this preprint. Click to download.

- Additionalfiles14.docx

- Additionalfiles5.pdf 